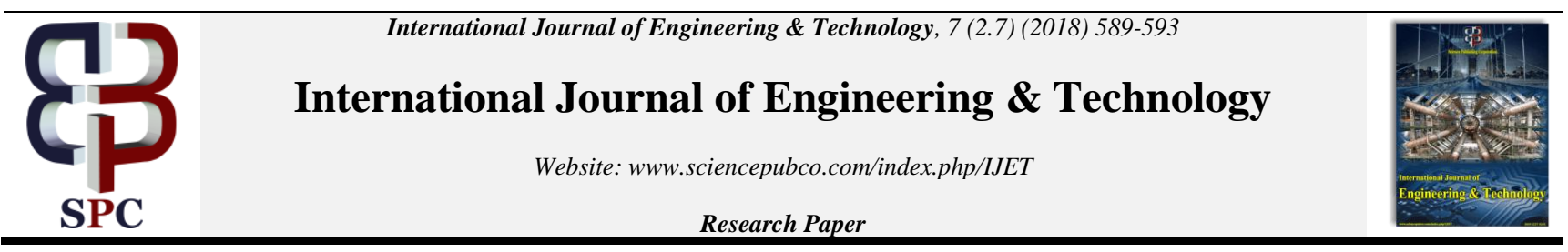

\title{
Sensor Enabled Wearable Technological Tool to Detect Breast Abnormality
}

\author{
M Kavitha ${ }^{1 *}$, P Venkata Krishna ${ }^{2}$ \\ ${ }^{I}$ Research Scholar, Dept of Computer Science, Sri Padmavati Mahila Visvavidyalayam, Tirupati \\ ${ }^{2}$ Professor, Dept of Computer Science, Sri Padmavati Mahila Visvavidyalayam, Tirupati \\ *Email: modepalli.kavitha@kluniversity.in
}

\begin{abstract}
Our Historical records say that, Women are back bone to every family. Generally we observe that health risk rate in women increases depends on age. Statistical medical reports proven that, early identification of health risks will increase the survival rate of patient. From last sixty years, we heard that breast cancer is one of the big health dare for women after forty years of age. Early phase detection of breast tumor is a big research issue in both developed and developing countries. Due to the limits in existing breast abnormality detecting tools like mammography, thermography, ultrasound, MRI there is a need to introduce smart, cost effective, patient comfortable, nonionic radiated compact testing tool to detect breast abnormality at early stage. In this work I am going to propose an advanced sensor enabled wearable technological tool design to detect breast abnormality. This tool is portable, patient comfortable, light weight, cost effective and suitable for dense breast, pregnant women and surgically altered breasts, younger women also.
\end{abstract}

Keywords: Breast cancer; Mammography; MRI - Magnetic resonance imaging; Thermography; Sensor enabled wearable technological tool.

\section{Introduction}

Breast Tumor still affects a bulky percentage of womenfolk in both developed and developing civilizations [1] and it is second driving reason of death for ladies. Breast tumor means a mass of tissue causes from uncontrolled grow and division of cells in a breast part of the body. If the uncontrolled growing cells act like usual cells, then the tumor is favorable (benign or not cancerous). If they are not act like usual cells and start to attack other soft tissue then the tumor is dangerous (cancerous or malignant). Like diverse diseases, tumor can attack and develop into the tissue encompassing the breast. That implies it can go to various parts of the body and shape new tumors, the method called metastasis.

The symptoms of breast tumor are a migraine or agony, a rash or swelling, thickened tissue in the woman's breast, or a lump, nipple discharge or change in appearance, breast size or shape change, skin temperature change of breast. Early identification and suitable treatment of breast cancer altogether increment the odds of existence [2]. The available breast tumor analytical methods are mammography, clinical inspection, thermography, ultrasound, MRI and biopsy etc. The methods were tried exclusively and in blends. In paper [3], Berg et al. presumed that that the most noteworthy affectability (99\%) was accomplished by joining finding from mammography, clinical examination, and MRI to the detriment of lower specificity (7\%). The general exactness of consolidating mammography, clinical examination, and MRI was not higher than the utilization of mammography alone (only $70 \%$ in the two cases). From Dr. Sila Yitta Radiologist of El Camino Hospital, self-screening and sporadic mammograms are the best safeguard, to detect breast abnormality at early stage. Due to ionic radiation, these imaging modalities are not preferable to performing scans frequently [18].

The aim of this paper is to develop a breast abnormality detecting tool that can be used with ease by patients to perform frequent periodic scans. The tool must thus be patient comfortable and compact.

\section{Associated effort}

Study on medical advancements in healthcare systems states that from past two eras sensors play a vital role in continuous monitoring of patient heath conditions. Today we get cost effective, noninvasive sensors to monitor the physical and mental changes of patient in market. Because of quick headways in low-control detecting, figuring, and correspondence, battery-fuelled Wireless Medical Sensors are ending up progressively universal.

According to Business Insiders explosion, in 201556 million plus wearable sensors were sold globally. So the number of wearable sensors sold rate expected to be 123 million by 2018 [9]. These devices catch, store, and convey physiological statistics unpretentiously, successfully, and efficiently. The summary of collectable physiological signs fuse, however isn't constrained to, heart frequency, body hotness, breath rate, circulatory strain, electroencephalogram, electrocardiogram, Galvanic skin reaction, oxygen immersion, blood glucose, and weight file $[10,11]$. Skin temperature is additionally quantifiable using a computerized temperature sensor [13]. This rundown is growing quickly, given the speed of continuous innovative headways in this field [12]. 
Wireless medical sensors can gather physiological signs from anybody anyplace whenever. Advances in current innovation have considered the improvement of scaled down remote portable wellbeing observing frameworks, equipped for nonstop checking while at the same time being power efficient. Need to design advanced gadgets using wearable body sensors to account the discovery of variations from the norm, unexpected circumstances and even give expectancy, all in view of the checking of physiological parameters in conjunction with conceivable side effects of symptoms [13]

From last 60 years breast malignant tumor is the mutual deadly infection among womenfolk throughout the world. Studies have presumed that early discovery and proper treatment of infection will expand the survival rate [2]. Breast growth is less demanding to treat, with less dangers and diminishes mortality by $25 \%$ when breast abnormality detected at early stage.

Mammography is a widely used imaging tool to detect breast abnormality at early stage instead of other techniques thermography, clinical examination, MRI, biopsy etc. Mammography is a lowmeasurement X-beam methodology that permits perception of the inside structure of the breast i.e irregularities like masses, microcalsifications and architectural misrepresentations $[14,15]$. If the women having breast cancer family history, according to doctors suggestion she need to attend periodic mammography. In this test directly X-rays are passing on breast so may causes splitting of existing tumor or it may causes other health problems and periodic mammography is not easy to middle class people due to its expensiveness. Due to this ionic radiation periodic mammogram is not preferable. Mammogram does not give good results for the women having dense breast. Paper [16] shows the lab view of mammography test procedure. To get clear internal structure, the breast is pressed in between flat surface plates. To adjust the breast position correctly on screening machine, the breast is touched and pushed by clinical director during test. Breast portion of women body is very sensitive so women may feel pain or discomfort during test. Due to ionic radiation mammography is not effective for females with dense breast, surgically altered breasts, pregnant women, younger women $[5,6]$. From this study we conclude that mammography is not suitable for all categories of women and it is a patient uncomfortable tool.

From medical studies, Tumor doesn't attack both breasts at a time and the temperature is distributed to both breasts proportionally. For normal women the temperature is expected to be symmetrical, with any variance less than 0.2 degrees $C$ [17]. From the medical facts, to sustain existing tumor, new blood vessels are formed due to that the temperature of breast part increases. So the alteration of temperature pattern in breasts is one of the key metric to find breast abnormality. The imaging technique thermography shows the temperature asymmetry of breasts [4]. That means thermography simply gives the temperature pattern of breasts. A little anomaly in temperature arrangement of left and right breast may adjust as breast irregularity from this test. But to attempt this test patient need to take some precautions to reduce the error rate in test $[7,8]$. If the tumor is deeper, that means not near to the skin then the temperature changes are not identified in this test. Women breast temperature will vary due to other reasons like menstrual cycle, teenage years, pregnancy, hormone replacement therapy, menopause, some gynecological diseases etc. In thermography procedure also the patient needs to place the chest part of body in front of the camera exactly to get right results[22-27]. For this the radiologist or clinical director will direct and touch if need the patient, to stand exactly in front of infrared rays. From this study we conclude that thermography is not efficient for all categories of women and it is also a patient uncomfortable tool.

Magnetic resonance imaging (MRI) uses an exceptional alluring field, radio repeat beats and a PC to make unequivocal pictures of organs, fragile tissues, and bone in every way that really matters all other inside body structures. Latest reports designate that MRI can distinguish tumor in the contralateral breast notwithstanding at the point when such developments were missed by mammography or in clinical examination at the time of the fundamental chest examination. What's more, MRI has been turned out to be a superior showing device for ladies with a solid family history of breast tumor. In spite of the way that the affectability of MRI is superior to that of mammography, the framework is blemished by a lower specificity and a far more noteworthy cost. The biopsy is the most exact system for confirming breast tumor and its stages since it is an immediate investigation of the living tissue. Be that as it may, it is tedious and requires convoluted methods.

This examination has inspired the momentum research to explore the new tool that could lead later on to an enhanced breast cancer detection modality. That means there is an urgent clinical need for a portable, practical, patient comfortable, non-ionic radiated and cost-effective device to detect early breast abnormality which can be universally applied to all patients. The objective of this study is to understand the advancements of sensors in patient health monitoring, and patient's views on breast abnormality screening tools - mammography, thermography etc. In this paper I am going to propose an advanced, sensor enabled, portable, practical, patient comfortable, non-ionic radiated and cost-effective wearable technological tool to detect breast abnormality at early stage.

\section{Projected effort: Design of sensor enabled wearable technological tool}

Through the use of sensor-based wearable innovation, client side effects can be checked, investigated and analyzed easily and fast. As part of my proposed work, to get breast health information or breast images we are going to place the sensors very close to the chest part of body. If we attach the sensors directly to the chest, it may cause side effects. So I am going to propose a bra (sleeveless jacket) type jacket for designing a sensor enabled tool. A bra is a form-fitting undergarment designed to support or covers the wearer's breasts. It is very close to the chest and in today's life style for fashion or culture names every woman uses this jacket in regular life [19]. If we design any shirt type jacket that is not close to the chest part and elder women may not feel comfortable. But bra is just like sleeveless jacket so it is very comfortable to all ages of women. To find breast abnormality I am proposing a Bra type sensor enabled wearable technological tool. In my proposed bra type tool, if the hook of the bra is at back side the older women needs the help from others to fix the hook while test. To avoid this I am proposing front hook type bra or bra without hooks. Fig 1 shows proposed jacket structure.

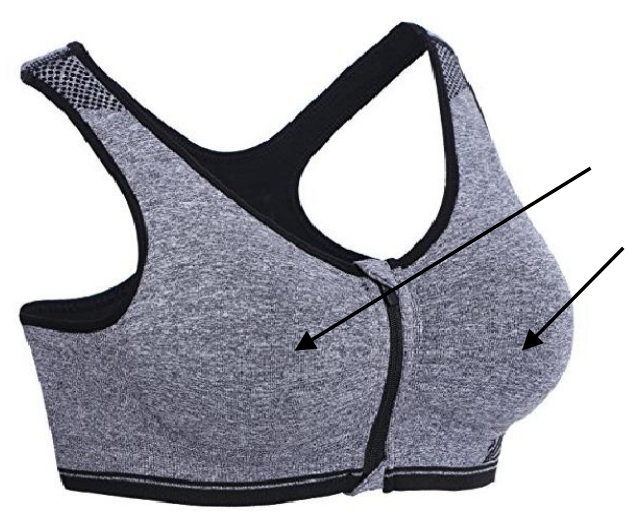

Fig. 1: Spandex fabric material Jacket and arrow marks shows sensor embedded patch position

To adjust to all categories of women I supposed stretched type of cloth to design the tool. Spandex is the ultimate stretch fab- 
ric material. This fabric material is compatible to all types of sensors and breathable, lightweight, wicking, quick drying. Due to the stretch mechanism of fabric material the designed jacket is comfort to all categories of women.

As part of this tool design, I proposed a small sensor embedded patch to stich inside the jacket. In figure 1 arrow marks shows the position to stich the patch. Inner side of jacket this proposed patches will be stitched to scan the breast health information. To design patch instead of using one sensor I am proposing 6 wireless sensors like intelligent bra patch proposed by Rob Royea, CEO of Cyrcadia Health and intelligent brasseries proposed in Keith et.al research work [21]. The tissue may form in any place of the breast. If we place all sensors at one place we are not able to get results exactly. The sensors are set on the best zones of enthusiasm on the bosom, in view of where most diseases create. In this work, the six wireless sensors are arranged in all sides of patch like one sensor in the middle, two at bottom and remaining three are at left, right and top side of patch. That means we are predefining the positions of all sensors. Additionally one microcontroller is placed in patch to control the operation of jacket. Like this structure we designed two wireless sensor embedded patches. Figure 2 shows wireless sensor embedded patch structure. In that red circles refer sensors. The square shape refers microcontroller. After, these patches are stitched over bra. Here we are using two patches in our proposed tool. So using this tool we can get the both breast health information at a time unlike imaging technology mammography.

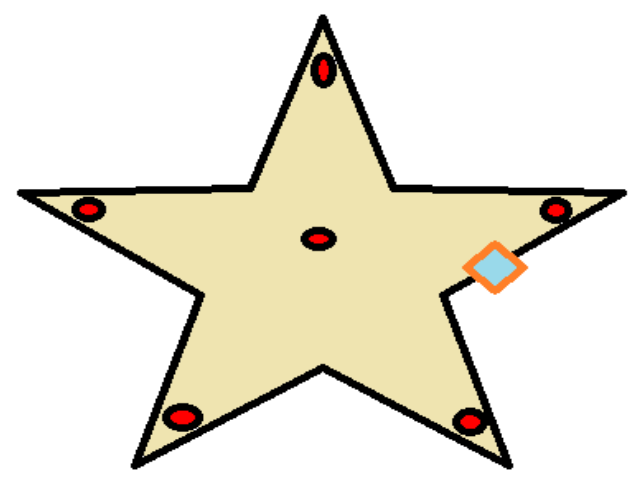

Fig. 2: Wireless sensor embedded patch design, circle refers sensor and square refers micro controller

A wide variety of sensors are available in market to support this tool design and different types of metrics are there to suspect breast tumor. Depends on our proposed metric we are going to select corresponding sensors to design wireless patch. So I am surveying different types of metrics to find breast abnormality.

1. From thermography tool we conclude that temperature asymmetry of breast is one of the metric to suspect breast tumor. Compare to normal cells, malignancy cells are developing and increasing quick so blood stream and digestion are higher in current tumor. As blood stream and digestion builds at that point skin temperature goes up. But if tumor is formed few millimeters deeper then surface blood flow doesn't change and women breast temperature will vary due to other reasons also like menstrual cycle, teenage years, pregnancy, hormone replacement therapy, menopause, some gynecological diseases etc. If the women works at outside due to work pressure or due to sun radiation also the temperature of breast will vary. So temperature abnormality alone is not a metric to suspect breast tumor in my view. So I am not considering temperature sensors to design the jacket.
2. From medical reports breast Swelling is one of the metric to suspect breast tumor. Human skin having stretchable capability. Due to stress or at the time of hormonal replacement the human skin will expand. Breast swelling is common in younger women at the time of menstrual cycle and for pregnant women also the breast size will vary. So I am not considering swelling detected supporting sensors to design the jacket.

3. From doctor's point of view breast portion skin redness, infection (rashes), nipple discharges other than milk are also some metrics to find tumor. Now a day, tight dress maintenance is fashion especially for females. Due to those tight dresses, sweat formed under breast part of skin. Due to this sweat, the skin irritation, redness, rashes are commonly occurs. Because of stress or at the time of hormonal replacement nipple discharge occurs some times. So I am not considering these metrics and supporting photo sensors to design the jacket.

4. ECG abnormality is also one of the metric to find tumor. Due to other health causes also we get abnormal ECG report. So I am not considering this metric and supporting ECG supported sensors to design the jacket.

5. Internal perception of breast is one of the metric to find tumor. Internal perception Shows internal structure of breasts like masses, microcalsifications and architectural misrepresentations. So I considered this as a supported metric and advanced photo sensors to get the internal perception of breast as a result.

\subsection{Projected tool working}

The Proposed tool is support to patients at laboratory or from home itself. Depends on the place it is used we need to enhance the design of microcontroller.

Figure 3 shows the structure of proposed system block diagram. The wireless sensors are configured depends on our requirements while designing the tool. In laboratory, the room temperature is adjusted to 22 degrees Celsius to relax and cool the patient body. After 5 to 10 minutes, the patient body will adjusted to room temperature and she wears this jacket. Human skin contains an extensive variety of layers including the epidermis, dermis, subcutis and a gathering of nerves, fibers, and fat. The dielectric properties of the skin have been represented and depicted by the professional in paper [20].Due to dielectric properties of human cell; the sensors in a jacket are activated. After All the sensors in a patch will send the analog data to microcontroller storage area. The microcontroller has the logic to convert this analog data into digital form and send to data analyst. Through Wi-Fi the data will reaches to radiologist system for analysis.

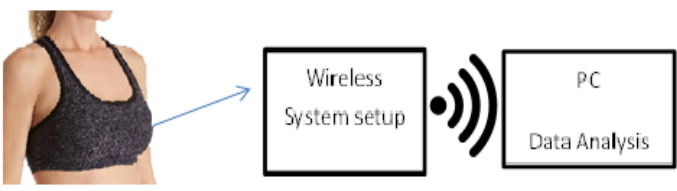

Fig. 3: Proposed system Block diagram

At radiologist system all processing steps done like image preprocessing, segmentation, feature extraction, and feature Classification using existing Computer Aided Design (CAD) techniques to find the breast abnormality. In other tests like mammography and thermography the radiologist or clinical director will help the patient to adjust the breast in right position. But in our proposed tool 
patient does not expect others help to take the test. Because wearing this jacket is comfort to patient.

To support the tool from home itself, we need enhance the microcontroller functionality. In laboratory supported tool the microcontroller taking the analog data from sensors, converts it into digital form and simply send to radiologist system. But in home-held tool, the microcontroller itself needs to take care of data processing also. As processing part, a framework for dissecting an arrangement of picture interpretations of bosom tissue of a topic to distinguish doubtful tissue and non- doubtful tissue, including: storage system- it stores the set of image readings gathered from a set of advanced photo sensors set at foreordained position on bosom of the subject, and an ensemble of classifier frameworks that are readied using no less than one genuine bosom picture readings including ordinary, strange and odd picture readings identified with suspect and non-suspect tissues, the ensemble of classifier systems receiving the set of image interpretations as an input, producing two or more output labels recognizing the breast tissue as speculate tissue or non-suspect tissue, and combining the two or more output labels into a single diagnosis recognizing the breast tissue as doubtful tissue or non- doubtful tissue, wherein a weight of each label from the two or more output labels determines a relative contribution of the label towards the single diagnosis. After analyzing part over, the microcontroller will send data to attendant or patient itself or personal doctor, that depends on the contact information specified at the time of tool design. For permanent recording of results, the system will send the data to cloud if mention that feature in our tool. Due to that permanent record of results it is possible to compare with previous reports also directly. This tool is very flexible to design.

\section{Analysis}

Existing imaging tools like mammography, thermography are not comfortable where as our proposed tool is very comfortable and easy to use by patients. Due to ionic-radiation of mammography frequent scans is not advisable. But the tumor family history patients need to attend the test periodically to detect the tumor at early stage. Our proposed tool supports frequent scans due to nonionic nature of sensors. Due to advancements in sensor field, we get sensors at very reasonable cost. So the test cost will affordable to all categories of patients. Due to the non-ionic nature of sensors our tool supports for dense breast, pregnant women, surgically altered breasts also unlike mammography. It is portable because of its size. The test using our tool will take less time compare to existing imaging tools like mammography and thermography. The patient doesn't require others help while taking test where as in mammography and thermography the patient needs the help of clinical director to adjust the breast correctly at machine. The material and components weight used to design this tool is very less, so our tool will be a light weight tool and it is portable.

\section{Conclusion}

In medical science, wireless medical sensors play a vigorous role in monitoring physical and mental changes of patient at remote location. In medical field, this proposed sensor enabled wearable technological tool will serve as more compare to existing breast abnormality detecting tools like mammography, thermography etc. This proposed breast abnormality detecting tool is portable, user comfortable, light weight, cost effective and suitable for dense breast, pregnant women and surgically altered breasts also.

\section{Future work}

To apply the sensor enabled wearable technological tool in real world, some challenges are there in our hand. I am going to describe one by one.
1. Need to identify or design the sensors suitable to get the internal perception of breast.

2. Need to generate best algorithms to process data and to find breast abnormality.

\section{References}

[1] Sfakianakis, Stelios, et al. "On the identification of circulating tumor cells in breast cancer." IEEE Journal of Biomedical and health informatics 18.3 (2014): 773-782.

[2] Hela, Boulehmi, et al. "Breast cancer detection: A review on mammograms analysis techniques." Systems, Signals \& Devices (SSD), 2013 10th International Multi-Conference on. IEEE, 2013.

[3] Berg, Wendie A., et al. "Diagnostic accuracy of mammography, clinical examination, US, and MR imaging in preoperative assessment of breast cancer." Radiology 233.3 (2004): 830-849.

[4] Gogoi, Usha Rani, et al. "Breast abnormality detection through statistical feature analysis using infrared thermograms." Advanced Computing and Communication (ISACC), 2015 International Symposium on. IEEE, 2015.

[5] Qi, Hairong, and Nicholas A. Diakides. "Thermal infrared imaging in early breast cancer detection." Augmented Vision Perception in Infrared. Springer, London, 2009. 139-152.

[6] Qi, Hairong, and Nicholas A. Diakides. "Thermal infrared imaging in early breast cancer detection-a survey of recent research." Engineering in Medicine and Biology Society, 2003. Proceedings of the 25th Annual International Conference of the IEEE. Vol. 2. IEEE, 2003.

[7] Wakankar, Asmita, G. R. Suresh, and Akshata Ghugare. "Automatic Diagnosis of Breast Abnormality Using Digital IR Camera." Electronic Systems, Signal Processing and Computing Technologies (ICESC), 2014 International Conference on. IEEE, 2014.

[8] Shahari, Sheetal, and Asmita Wakankar. "Color analysis of thermograms for breast cancer detection." Industrial Instrumentation and Control (ICIC), 2015 International Conference on. IEEE, 2015.

[9] Yin, Hongxu, and Niraj K. Jha. "A Health Decision Support System for Disease Diagnosis Based on Wearable Medical Sensors and Machine Learning Ensembles." IEEE Transactions on Multi-Scale Computing Systems 3.4 (2017): 228-241.

[10] Baig, Mirza Mansoor, and Hamid Gholamhosseini. "Smart health monitoring systems: an overview of design and modeling." Journal of medical systems 37.2 (2013): 9898.

[11] Lee, Hyunjae, et al. "A graphene-based electrochemical device with thermoresponsive microneedles for diabetes monitoring and therapy." Nature nanotechnology 11.6 (2016): 566

[12] Majumder, Turbo, et al. "NoC-enabled multicore architectures for stochastic analysis of biomolecular reactions." Design, Automation \& Test in Europe Conference \& Exhibition (DATE), 2015. IEEE, 2015.

[13] Wannenburg, Johan, and Reza Malekian. "Body sensor network for mobile health monitoring, a diagnosis and anticipating system." IEEE Sensors Journal 15.12 (2015): 6839-6852.

[14] Islam, Md Shafiul, Naima Kaabouch, and Wen Chen Hu. "A survey of medical imaging techniques used for breast cancer detection." Electro/Information Technology (EIT), 2013 IEEE International Conference on. IEEE, 2013.

[15] Paramkusham, Spandana, Kunda MM Rao, and BVVSN Prabhakar Rao. "Early stage detection of breast cancer using novel image processing techniques, Matlab and Labview implementation." Advanced Computing Technologies (ICACT), 2013 15th International Conference on. IEEE, 2013.

[16] Yin, Hongxu, and Niraj K. Jha. "A Health Decision Support System for Disease Diagnosis Based on Wearable Medical Sensors and Machine Learning Ensembles." IEEE Transactions on Multi-Scale Computing Systems 3.4 (2017): 228-241.

[17] Potter, Helen, et al. "The sensitivity of thermography to temperature changes in breast tissue." Australian Journal of Physiotherapy 43.3 (1997): 205-210.

[18] El Hallaoui, Karim, et al. "Signal analysis and phantom experiments for a miniaturized time-domain microwave breast health monitoring device." Antennas and Propagation (EUCAP), 2017 11th European Conference on. IEEE, 2017 
[19] Kim, Sun Young. "Expression Trend and Characteristics of Pixel in Contemporary Fashion." Korean Journal of Human Ecology 24.3 (2015): 407-421.

[20] Gabriel, S., R. W. Lau, and Camelia Gabriel. "The dielectric properties of biological tissues: II. Measurements in the frequency range $10 \mathrm{~Hz}$ to $20 \mathrm{GHz}$." Physics in medicine \& biology 41.11 (1996): 2251.

[21] Keith, Louis G., et al. "System for analyzing thermal data based on breast surface temperature to determine suspect conditions." U.S. Patent No. 8,231,542. 31 Jul. 2012.

[22] Kishore, P.V.V., Kishore, S.R.C. And Prasad, M.V.D. 2013. Conglomeration Of Hand Shapes And Texture Information For Recognizing Gestures Of Indian Sign Language Using Feed Forward Neural Networks. International Journal Of Engineering And Technology, 5(5), Pp. 3742-3756.

[23] Varun Teja, T. And Asadi, S.S., 2016. An Integrated Approach For Evaluation Of Environmental Impact Assessment - A Model Study. International Journal Of Civil Engineering And Technology, 7(6), Pp. 650-659.

[24] Lakshmi, M.L.S.N.S., Khan, H. And Madhav, B.T.P. 2015. Novel Sequential Rotated $2 \times 2$ Array Notched Circular Patch Antenna. Journal Of Engineering Science And Technology Review, 8(4), Pp. 73-77.

[25] Jawahar, A. And Koteswara Rao, S., 2015. Recursive Multistage Estimator For Bearings Only Passive Target Tracking In Esm Ew Systems. Indian Journal Of Science And Technology, 8(26),

[26] Sarma, G.S., Asadi, S.S. And Narayana, S.L., 2016 Creation Of Web Based Decision Support Information System For Evaluation Of Topographic Characteristics Using Remote Sensing \& Gis And Visual Basic Programe. International Journal Of Civil Engineering And Technology, 7(6), Pp. 621-634.

[27] Aditya Varma, K.V., Manideep, T. And Asadi, S.S., 2016. A Critical Comparison Of Quantity Estimation For Gated Community Construction Project Using Traditional Method Vs Plan Swift Software: A Case Study. International Journal Of Civil Engineering And Technology, 7(6), Pp. 707-713. 